\title{
On the widths of the $\mathrm{Mg} \mathrm{X}$ lines near $60 \mathrm{~nm}$ in the corona
}

\author{
K. Wilhelm ${ }^{1}$, B. N. Dwivedi ${ }^{1,2}$, and L. Teriaca ${ }^{1}$ \\ 1 Max-Planck-Institut für Aeronomie, Max-Planck-Str. 2, 37191 Katlenburg-Lindau, Germany \\ 2 Department of Applied Physics, Banaras Hindu University, Varanasi-221005, India
}

Received 27 August 2003 / Accepted 3 November 2003

\begin{abstract}
We examine the line widths of both components of the $\operatorname{Mg~X} 2 \mathrm{~s}^{2} \mathrm{~S}_{1 / 2}-2 \mathrm{p}^{2} \mathrm{P}_{1 / 2,3 / 2}$ doublet at $60.98 \mathrm{~nm}$ and $62.50 \mathrm{~nm}$ in the low corona of the quiet Sun, and find that the Doppler width (i.e. half $1 / e$ width) broadens from $\Delta \lambda_{\mathrm{D}} \approx 8.2 \mathrm{pm}$ to $\approx 9.5 \mathrm{pm}$ (with an estimated relative standard uncertainty of $4 \%$ ) between the limb and $220 \mathrm{Mm}$ above the limb in the equatorial corona. In a polar coronal hole, the Doppler width increases from $10.8 \mathrm{pm}$ near $30 \mathrm{Mm}$ to $11.4 \mathrm{pm}$ at around $80 \mathrm{Mm}$. The analysis does not provide any evidence for a narrowing of the emission-line profiles as a function of the distance from the solar limb.
\end{abstract}

Key words. Sun: corona - Sun: UV radiation

\section{Introduction}

Harrison et al. (2002) reported the narrowing of the $\mathrm{Mg}$ X $62.50 \mathrm{~nm}$ line with height in the quiet near-equatorial solar corona, thereby concluding that this narrowing is most likely evidence of dissipation of Alfvén waves in closed fieldline regions. Similarly, a significant change in slope of the line width as a function of height was seen in polar coronal holes by O'Shea et al. (2003) at an altitude of $\approx 65 \mathrm{Mm}$. These results obtained with the Coronal Diagnostic Spectrometer (CDS) (Harrison et al. 1995), if confirmed, could be of the utmost importance in understanding the mechanisms that heat the corona. Due to the broad instrumental profile, the CDS instrument can only study line-width variations and cannot provide measurements of the line width itself, and, hence, of the effective ion temperature. Since the latter quantity is critical in constraining theoretical models of coronal heating and solar-wind acceleration, for instance, through the dissipation of high-frequency waves generated by chromospheric reconnection, we study the problem further by analysing data recorded with the Solar Ultraviolet Measurements of Emitted Radiation (SUMER) spectrograph in the $\mathrm{Mg} \mathrm{X}$ doublet together with other neighbouring lines in both the quiet equatorial corona and in a polar coronal hole. Due to the high spectral resolution of SUMER, we are able to obtain profiles of both Mg X emission lines and measure their widths and variations as a function of height.

For a line profile of Gaussian shape, the Doppler width, $\Delta \lambda_{\mathrm{D}}$, is related to the standard deviation, $\sigma$, and the full width at half maximum, $\Delta \lambda_{\mathrm{FWHM}}$, through

$\Delta \lambda_{\mathrm{D}}=\sigma \sqrt{2}=\Delta \lambda_{\mathrm{FWHM}} /(2 \sqrt{\ln 2})$.

Send offprint requests to: $\mathrm{K}$. Wilhelm, e-mail: wilhelm@linmpi.mpg.de
Assuming a Maxwellian velocity distribution of the photonemitting ions of mass $m_{\mathrm{i}}$ and temperature $T_{\mathrm{i}}$, a relation between $\Delta \lambda_{\mathrm{D}}$ and $T_{\mathrm{i}}$ can be obtained (cf., e.g. Mariska 1992)

$\Delta \lambda_{\mathrm{D}}=\frac{\lambda_{0}}{c_{0}} \sqrt{\frac{2 k T_{\mathrm{i}}}{m_{\mathrm{i}}}}$

where $\lambda_{0}$ is the rest wavelength, $c_{0}$ the speed of light in vacuum, and $k$ the Boltzmann constant. Equation (2) describes the thermal width of a spectral line. Brueckner \& Moe (1972) and others found line widths in the solar atmosphere in excess of the thermal broadening expected from a temperature at the maximum of the ionic fraction. This led to the introduction of a nonthermal velocity contribution, $\xi$, and (for Gaussian profiles) to a total Doppler width of

$\Delta \lambda_{\mathrm{D}}=\frac{\lambda_{0}}{c_{0}} \sqrt{\frac{2 k T_{\mathrm{i}}}{m_{\mathrm{i}}}+\xi^{2}}=\frac{\lambda_{0}}{c_{0}} \sqrt{\frac{2 k T_{\mathrm{i}, \mathrm{eff}}}{m_{\mathrm{i}}}}=\frac{\lambda_{0}}{c_{0}} v_{1 / e}$.

$T_{\mathrm{i}, \mathrm{eff}}$ is defined as effective ion temperature and $v_{1 / e}$ is the mostlikely speed along the line of sight (LOS).

The separation into thermal and non-thermal contributions and the physical interpretation of the non-thermal broadening have been discussed in many recent articles on the plasma conditions of the solar atmosphere (e.g. Seely et al. 1997; Teriaca et al. 2003). We will not attempt to suggest any improvements on this separation here. However, we would like to address the more basic problem of deducing the total line width in the corona from recent observations. Specifically, we will determine the line widths of the $\mathrm{Mg} \times 2{ }^{2} \mathrm{~S}_{1 / 2}-2 \mathrm{p}^{2} \mathrm{P}_{1 / 2,3 / 2}$ doublet at $60.98 \mathrm{~nm}$ and $62.50 \mathrm{~nm}$. The best wavelength determinations ${ }^{1}$ for all potentially relevant lines near the $\mathrm{Mg} \mathrm{X}$ doublet

\footnotetext{
${ }^{1}$ In the text we use rounded values sufficient to identify the lines.
} 
Table 1. The components of the lithium-like $\operatorname{MgX}$ doublet and the emission lines in their neighbourhoods.

\begin{tabular}{|c|c|c|c|c|}
\hline \multirow[t]{2}{*}{ Lines } & \multirow{2}{*}{$\begin{array}{l}\text { Wavelength }^{a} \\
\lambda / \mathrm{nm}\end{array}$} & \multicolumn{2}{|c|}{ Radiances } & \multirow[t]{2}{*}{ Remarks } \\
\hline & & $L_{\mathrm{K}}^{b}$ & $L_{\mathrm{C}}^{c}$ & \\
\hline O IV & 60.8398 & 800 & 731 & outside Fig. 2 \\
\hline $\mathrm{OV}$ & 121.834 & - & - & \\
\hline O III & 60.9705 & 300 & 1.61 & blend \\
\hline $\operatorname{Mg} X$ & 60.9795 & 23 & 457 & blend \\
\hline O IV & 60.9829 & 850 & 1300 & blend $^{d}$ \\
\hline O III & 61.0043 & 350 & 5.26 & not visible \\
\hline $\mathrm{C}_{\mathrm{I}}$ & 124.9004 & 10 & - & \\
\hline O IV & 62.4617 & 750 & 3.27 & \\
\hline Si $\mathrm{X}$ & 62.470 & - & - & \\
\hline C I & 124.9405 & 20 & - & \\
\hline P II & 124.9812 & - & - & blend \\
\hline $\operatorname{Mg} X$ & 62.4965 & 12 & 227 & blend \\
\hline Mg II & 124.993 & - & - & blend \\
\hline Si II & 125.0089 & 100 & - & blend \\
\hline OIV & 62.5130 & 800 & 6.57 & blend \\
\hline C I & 125.0423 & 30 & - & \\
\hline Si II & 125.0433 & 150 & - & \\
\hline S II & 125.0578 & - & - & prominent \\
\hline Si II & 125.1164 & 200 & - & weak \\
\hline C I & 125.1176 & 40 & - & \\
\hline O IV & 62.5852 & 850 & 9.82 & weak \\
\hline
\end{tabular}

${ }^{a}$ For line identifications and wavelengths see Cohen et al. (1978); Sandlin et al. (1986); Kelly (1987); Dammasch et al. (1999); Peter \& Judge (1999), and Curdt et al. (2001). The first and second orders of diffraction are superimposed in SUMER spectra.

${ }^{b}$ Relative radiances after Kelly (1987); separately given for the different spectra listed with more than one line.

${ }^{c}$ Relative radiances derived from CHIANTI (cf. Dere et al. 1997) for isothermal plasmas at the formation temperature of each line and a density of $n_{\mathrm{e}}=1 \times 10^{9} \mathrm{~cm}^{-3}$.

${ }^{d}$ Relative radiance of O IV is $15 \%$ of $L(\mathrm{MgX})+L(\mathrm{O}$ IV $)$ at $29^{\prime \prime}$ to $80^{\prime \prime}$ above the limb.

and the doublet itself are compiled in Table 1. The first observation of the $\mathrm{Mg} \mathrm{X}$ line widths was performed by Hassler et al. (1990) on the solar disk and in the north-west corona out to distances of $1.2 R_{\odot}$ (solar radius) during a sounding rocket flight. They found a disk average of $\Delta \lambda_{\mathrm{D}} \approx 8.3 \mathrm{pm}$ for the $60.98 \mathrm{~nm}$ line $\left(v_{1 / e} \approx 41 \mathrm{~km} \mathrm{~s}^{-1}\right)$ and an increase to $11 \mathrm{pm}$ for both $\mathrm{Mg} \mathrm{X}$ lines at a tangent-ray height of $70 \mathrm{Mm}$ followed by a plateau to $140 \mathrm{Mm}$.

With the advent of the ESA and NASA Solar and Heliospheric Observatory (SOHO), many more measurements could be performed by its spectroscopic instruments. Using SUMER observations, Erdélyi et al. (1998) obtained $\Delta \lambda_{\mathrm{D}}=7.7 \mathrm{pm}$ for the $\mathrm{MgX} 62.50 \mathrm{~nm}$ line at the centre of the disk, and $\approx 8.2 \mathrm{pm}$ near the equatorial limb. (Note that first-order values of the widths of lines seen in the second order are listed in Table 1 of Erdélyi and co-workers). A $360^{\circ}$ roll manœuvre of SOHO on 20 March 1997 with stops every $30^{\circ}$ provided limb brightening and broadening information on spectral lines in the range from $58 \mathrm{~nm}$ to $140 \mathrm{~nm}$ (Dammasch et al. 1999). Pronounced broadenings near the limb were found for most of the transition-region lines, which were generally a little wider in coronal-hole than in quiet-Sun regions. The limb broadening of the $\mathrm{MgX} 62.50 \mathrm{~nm}$ line could, however, not be accurately determined because, in this sequence of observations, the line was observed on the potassium bromide (KBr) photocathode of detector $\mathrm{B}$ and was blended (in all likelihood by the PII, Mg II, Si II lines in the first order and possibly by the OIV $62.51 \mathrm{~nm}$ line in the second order; see Sect. 5). Off the limb, the line width was $\Delta \lambda_{\mathrm{D}}=7.1 \mathrm{pm}$. During inter-calibration observations in quiet regions of the disk, a value of $\Delta \lambda_{\mathrm{D}}=7.4 \mathrm{pm}$ was obtained for both $\mathrm{Mg} \mathrm{X}$ lines with SUMER on 2 November 1999 (Dammasch 2000, personal communication).

SUMER observations during another SOHO roll manœuvre on 21 and 22 November 1996 were analysed by Doschek $\&$ Feldman (2000). Near the equatorial plane, the values for the $\mathrm{Mg}$ X $60.98 \mathrm{~nm}$ line slightly increased from $8.2 \mathrm{pm}$ obtained in the height range $20 \mathrm{Mm}$ to $90 \mathrm{Mm}$ to $9.2 \mathrm{pm}$ between $210 \mathrm{Mm}$ and $310 \mathrm{Mm}$. Considering the uncertainties involved, the authors did not claim that a significant increase in line width with height had been found. Doschek et al. (2001) studied the line widths of the $\mathrm{MgX}$ doublet in a north-polar coronal hole in 1996. The results can be summarized (converted to Doppler widths and corrected for instrumental effects here) as a slow increase from $\Delta \lambda_{\mathrm{D}}=8.8 \mathrm{pm}$ to $10.1 \mathrm{pm}$ for $\mathrm{MgX} 60.98 \mathrm{~nm}$ over a height range from $14 \mathrm{Mm}$ to $85 \mathrm{Mm}$, and $\Delta \lambda_{\mathrm{D}}=8.1 \mathrm{pm}$ to $10.7 \mathrm{pm}$ for the $62.50 \mathrm{~nm}$ line from the limb to $71 \mathrm{Mm}$.

The narrowing of the $\mathrm{Mg}$ X $62.50 \mathrm{~nm}$ line with height in the quiet equatorial corona observed with CDS in 2001 (Harrison et al. 2002) could be interpreted as implying that there were significantly different conditions in quiet areas of the corona in 1996 (near the sunspot minimum) and 2001 (near the sunspot maximum). However, the CDS authors suggest that this effect had not been identified in the SUMER observations of 1996 because of large uncertainties in the line-width determinations. We, therefore, re-analyse the line-width variations observed with SUMER in undisturbed regions of the Sun for the early SOHO mission period.

\section{The SUMER spectrometer}

The SUMER instrument is described by Wilhelm et al. (1995) and its performance characteristics are outlined in the firstresult papers (Lemaire et al. 1997; Wilhelm et al. 1997). The spectral resolution element (one spectral detector pixel) is $2.15 \mathrm{pm}$ for second-order lines near $61 \mathrm{~nm}$, and the instrumental width for detector B and the narrowest slit of $0.3^{\prime \prime}$ is $\Delta \lambda_{\mathrm{I}}^{\mathrm{B}}=7.6 \mathrm{pm}$ in the first order (for detector $\mathrm{A}$ we have $\Delta \lambda_{\mathrm{I}}^{\mathrm{A}}=5.7 \mathrm{pm}$ ). Corrections of the line widths for instrumental effects can be performed with a de-convolution function taking into account the order of diffraction and the actual slit width (with a non-Gaussian profile) employed during the observations. The programme, con_width_funct_3 .pro, is available from the SolarSoft Library. In this paper, all widths given as $\Delta \lambda_{\text {FWHM }}$ include the instrumental broadening ${ }^{2}$, whereas the $\Delta \lambda_{\mathrm{D}}$ values are corrected for this effect.

\footnotetext{
2 With an exception in Eq. (1).
} 
Despite the good suppression of scattered radiation in the SUMER instrument, the weak coronal emission can be influenced by the bright solar disk, which illuminates the primary telescope mirror at all times, and adequate consideration of this aspect is required. Whereas for spectral lines with low formation temperatures, an assessment is relatively straightforward (see Hassler et al. 1997; Lemaire et al. 1998; Feldman et al. 1999), it is more difficult to separate the scattered contribution from the coronal emission for lines with high formation temperatures, such as the $\mathrm{Mg} \mathrm{X}$ doublet. The radiance scale height for $\mathrm{Mg} \mathrm{X}$ up to at least $210 \mathrm{Mm}$ is $H_{\mathrm{R}}=50 \mathrm{Mm}$, but the scatter scale height is approximately a factor of five larger in this range (Feldman et al. 1999). The scatter scale height above this range is even $H_{\mathrm{S}}=350 \mathrm{Mm}$.

\section{Observations and data reduction}

The SUMER observations considered here were obtained under quiet solar conditions during a $90^{\circ}$ roll manœuvre in November 1996, and above a well-developed south-polar coronal hole on 16 May 1997. The data reduction ${ }^{3}$ used the mostrecent standard SUMER software to produce height and line profiles in Figs. 1 to 4 . The brightest line profile has been adjusted to the nominal rest wavelength in each of the graphical presentations, because velocity measurements of the bulk solar plasma were not the topic of this study; nevertheless, it can be seen that there were no systematic variations with altitude. The height profiles have been generated by subtracting the continuum levels (indicated by diamond symbols in Figs. 2 to 4) from the spectra, and integrating the emission lines over a range of $\lambda_{0} \pm 15 \mathrm{pm}$. Considering that this corresponds to $\approx 2 \Delta \lambda_{\mathrm{FWHM}}$, $96 \%$ of the total line radiance is included. Therefore, we plot as height profiles $100 / 96$ of the radiance obtained by the integration. The instrumental width is of no importance here.

\section{Results}

Simultaneously with the $\mathrm{Mg}$ X lines, the N v line at $123.88 \mathrm{~nm}$ was recorded to define the precise limb position of the equatorial measurements. Its height profile near the limb is shown in Fig. 1 together with the $\mathrm{Mg} \mathrm{X}$ data. The figure also provides the ratio of the radiances, $L_{62.50} / L_{60.98}$, of the $\mathrm{MgX}$ doublet. It is close to 0.5 everywhere in the corona indicating that the excitation of the $\mathrm{Mg}^{9+}$ ion is collisionally dominated there. However, near the limb and on the disk, the ratio drops to about 0.4 . We attribute this change mainly to a blend of the $\mathrm{Mg}$ X $60.98 \mathrm{~nm}$ line by the O IV line at $60.98 \mathrm{~nm}$ (cf. Sect. 5). The radiation scale height of $H_{\mathrm{R}}=50 \mathrm{Mm}$ deduced from Fig. 1 over the height range from $10 \mathrm{Mm}$ to $200 \mathrm{Mm}$ implies that the influence of scattered radiation must be very small in our data below $100 \mathrm{Mm}$, where a narrowing of the line width was observed with CDS. For instance, a fractional contribution of $12 \%$ to the total would change the effective scale height from $50 \mathrm{Mm}$

${ }^{3}$ All the raw data of SUMER are in the public domain and can be obtained from

http://www.linmpi .mpg.de/english/projekte/sumer/FILE/ SumerEntryPage.html

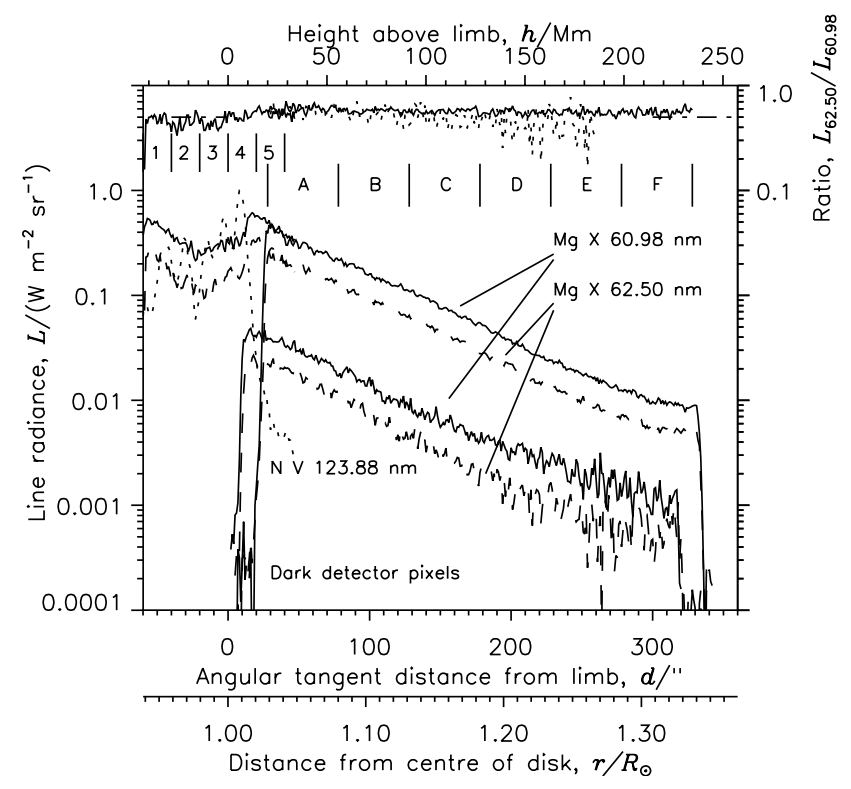

Fig. 1. Line radiances of the $\mathrm{MgX}$ doublet as a function of the radial distance in the equatorial plane (upper pair of graphs). The $\mathrm{NV} 123.88 \mathrm{~nm}$ height profile near the limb is also shown. The observations have been obtained at two different pointing positions (centre of slit) at $x_{\mathrm{S}}=970 "$; $y_{\mathrm{S}}=0 "$ in solar coordinates ( $x_{\mathrm{S}}$ : west; $y_{\mathrm{S}}$ : north) with a $0.3^{\prime \prime} \times 120^{\prime \prime}$ slit, and at $x_{\mathrm{S}}=1160^{\prime \prime} ; y_{\mathrm{S}}=0^{\prime \prime}$ with the $4^{\prime \prime} \times 300^{\prime \prime}$ slit. The radiances of the higher range had to be raised by a factor of 1.6 to achieve a smooth transition. A temporal change is the most likely cause of this variation (more than $2 \mathrm{~h}$ elapsed between the limb and the coronal observations). Some of the dark pixels of the detector have been shown to demonstrate the signal-to-noise margin. $\mathrm{Mg} \mathrm{X}$ radiances are also plotted above a south-polar coronal hole for a pointing positions of $x_{\mathrm{S}}=0^{\prime \prime}$ and $y_{\mathrm{S}}=-1142^{\prime \prime}$ (lower pair). The ratio, $L_{62.50} / L_{60.98}$, is plotted in the upper section of the diagram and compared with its expected value of $\approx 0.5$ (dashed-dotted line; equatorial ratio: solid line; polar coronal hole: dotted line). The intervals labelled 1 to 5 or A to $\mathrm{F}$ define the integration limits used to generate the spectral profiles in Figs. 2 to 4.

to $\approx 60 \mathrm{Mm}$, which can be excluded by the observations. We will demonstrate below that even a relative contribution of $20 \%$ has only a marginal impact on the line width under coronal conditions.

In Fig. 1, integration intervals are indicated for which detailed spectra near the $\mathrm{Mg} \mathrm{X}$ doublet are plotted in Figs. 2 and 3 for various height ranges. Finer height intervals did not lead to any new information, but cluttered the diagrams with additional profiles. When two spectra of the same wavelength range are available, their evaluations are separately documented in Table 2 to get an idea of the consistency of independent data points. The $\mathrm{Mg}$ X lines could be accurately fitted by Gaussian approximations. The largest relative deviation from any of the independent line width values is $\pm 5 \%$, and the relative standard deviation from the mean width at a certain altitude is $\approx 4 \%$. Of course, there may be additional systematic uncertainties, such as an inaccurate instrumental width used for obtaining the corrected Doppler width, but they are expected to affect all widths in the same manner and thus would not reverse any trend in a height profile. 


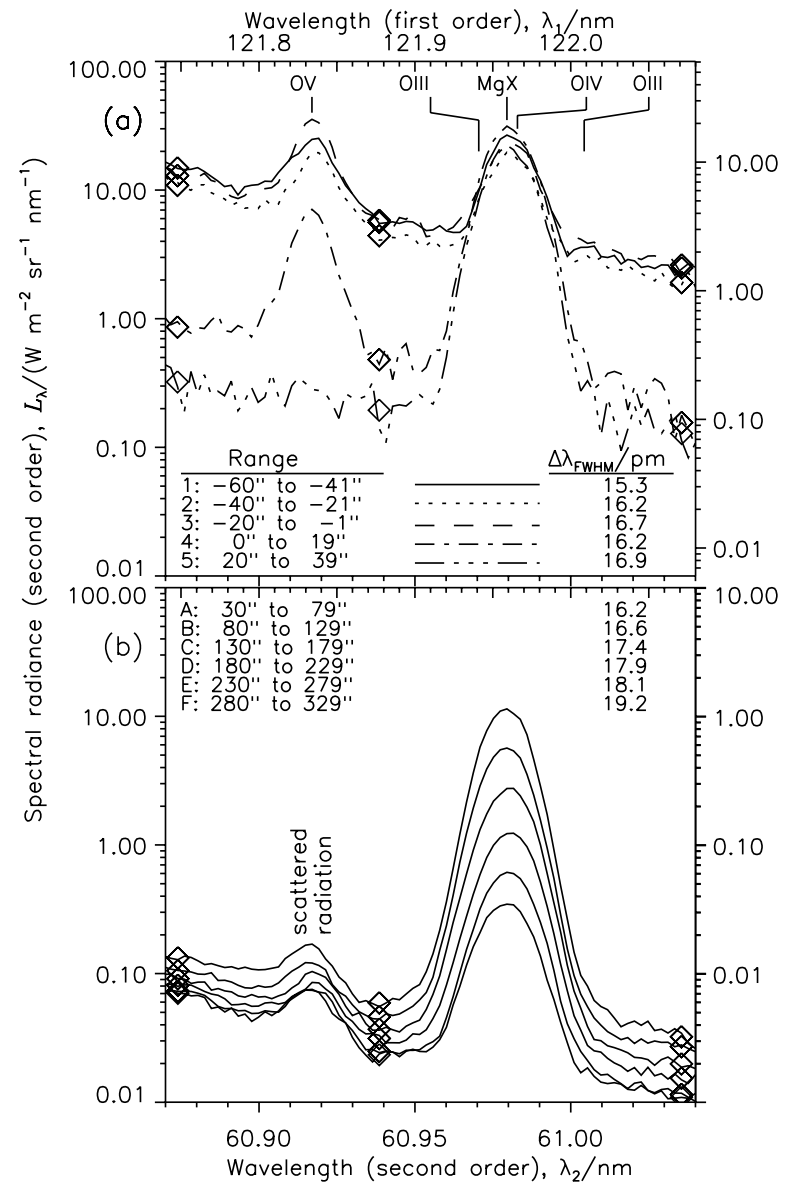

Fig. 2. a) The spectral radiance of the quiet Sun for wavelengths between 60.87 and $61.04 \mathrm{~nm}$. The $\mathrm{Mg}$ X $60.98 \mathrm{~nm}$ line in the second order and neighbouring lines are plotted for selected height ranges near the west limb (cf. Fig. 1). The O v $121.83 \mathrm{~nm}$ line and the background are seen in first order, for which the radiance scale is given on the right side. The data points determining the continuum are marked by diamond symbols. The line widths, $\Delta \lambda_{\mathrm{FWHM}}$, obtained by Gaussian fits, refer to the $\mathrm{Mg} \mathrm{X}-\mathrm{O}$ III-O IV blend and the corresponding height ranges. The $0.3^{\prime \prime} \times 120^{\prime \prime}$ slit and the bare detector B were used during the observation. b) The $\mathrm{Mg} \times 60.98 \mathrm{~nm}$ line profiles at greater heights. The observations were performed with the 4 " wide slit and the $\mathrm{KBr}$ photocathode.

Figures 2 and 3 show, in addition to the $\mathrm{Mg}$ X profiles, some other emission lines, most of them in the first order of diffraction. A relatively strong $\mathrm{C}$ I line at $124.94 \mathrm{~nm}$ and a Si X line at $62.47 \mathrm{~nm}$ in the second order are close to the $\mathrm{Mg} \mathrm{X}$ line. A pre-conditioned multi-Gaussian procedure allowed us to separate these lines from the $\mathrm{MgX}$ line quantitatively with results of $\approx 8 \%$ for the combined relative contributions of the $\mathrm{C} I$ and $\mathrm{Si} \mathrm{X}$ lines. The background is seen in the first order, namely the Ly $\alpha$ wing in Fig. 2 and the Si I continuum ${ }^{4}$ in Fig. 3.

As mentioned in Sect. 1, a slight increase of the line width was found for the $\mathrm{Mg}$ X $60.98 \mathrm{~nm}$ line in a polar coronal hole up to $\approx 85 \mathrm{Mm}$ by Doschek et al. (2001). The scale height between $20^{\prime \prime}$ and 140" above the limb for the $\mathrm{Mg}$ X $62.50 \mathrm{~nm}$ line was $50 \mathrm{Mm}$. We extend the observational range to about

\footnotetext{
${ }^{4}$ The relative contribution of the H I Lyman continuum in the second order is less than 5\% (cf. Wilhelm et al. 2002).
}

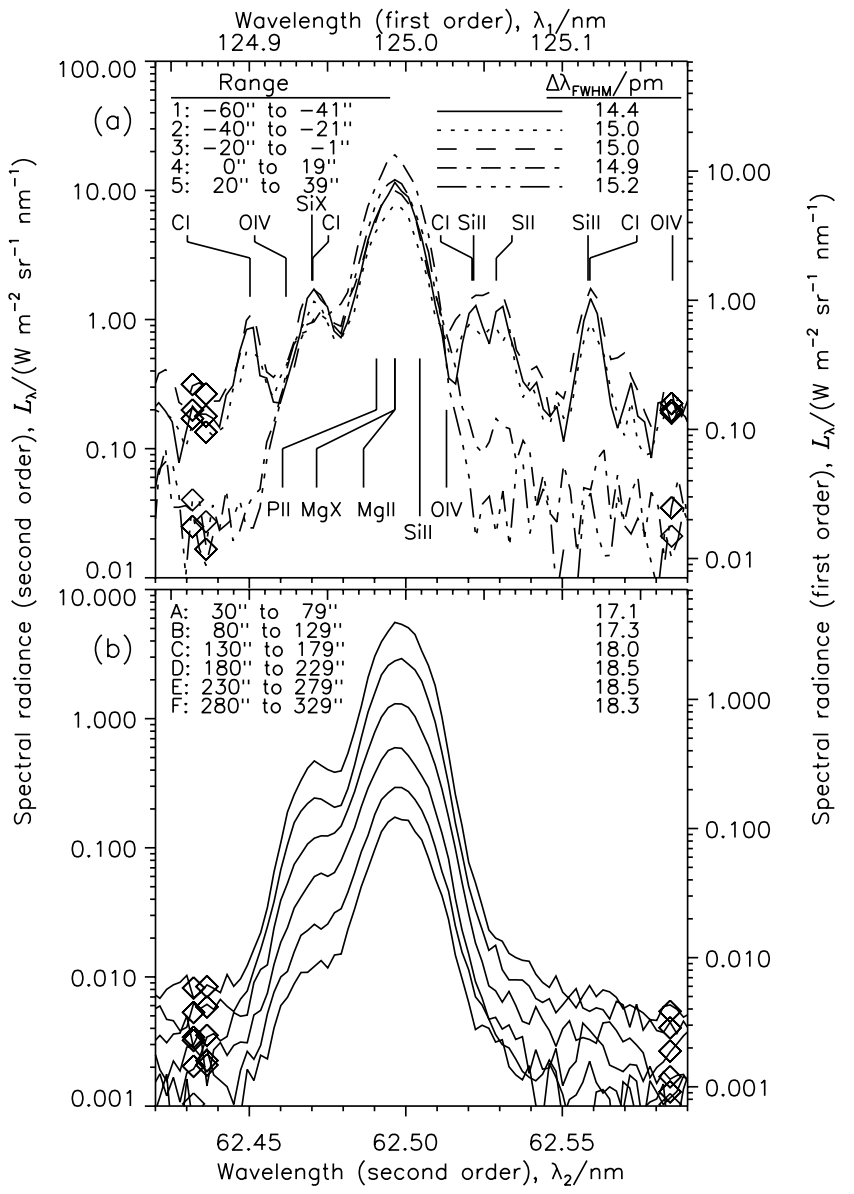

Fig. 3. a) The spectral radiance near the $\mathrm{Mg} X 62.50 \mathrm{~nm}$ line for height ranges close to the west limb (cf. Fig. 1). The $\mathrm{Mg} \mathrm{X}, \mathrm{Si}$ X and O IV lines are diffracted in the second order, all the other lines and the continuum are in first order. A few lines blend with the $\operatorname{MgX}$ line. The values of $\Delta \lambda_{\text {FWHM }}$ are given for the blend. A slit width of $0.3^{\prime \prime}$ and the bare portion of detector B were used. b) The $\mathrm{Mg} \mathrm{X} 62.50 \mathrm{~nm}$ and Si X $62.47 \mathrm{~nm}$ lines at greater heights observed with the 4 " slit and the bare detector.

$130 \mathrm{Mm}$, although the low emission in coronal holes causes severe difficulties. This altitude should be sufficiently high to cover the "turnover point" around $65 \mathrm{Mm}$ discussed by O'Shea et al. (2003). The line radiance of the Mg X doublet above a polar coronal hole is about a factor of ten lower than in the equatorial corona, as can be seen from Fig. 1. The scale height, however, is the same, namely $50 \mathrm{Mm}$ below a tangent distance from the limb of $180^{\prime \prime}$. Above this distance, it rapidly increases to $\approx 100 \mathrm{Mm}$. We attribute this increase to the influence of scattered radiation from the disk with a large apparent scale height. Consequently, we do not consider measurements above $\approx 180^{\prime \prime}$ as reflecting true coronal emission. This conclusion is supported by the ratio of the line radiances that, besides becoming very noisy, drops on average to disk values of $\approx 0.4$.

As for the equatorial observations, the polar coronal hole measurements had to be integrated over certain height ranges in order to achieve adequate counting statistics. Up to a tangent height of $180^{\prime \prime}$, we used the ranges indicated in Fig. 1, but combined ranges $\mathrm{D}$ to $\mathrm{F}$ to obtain a relative smooth profile that is most likely affected by scattered radiation from the disk. 


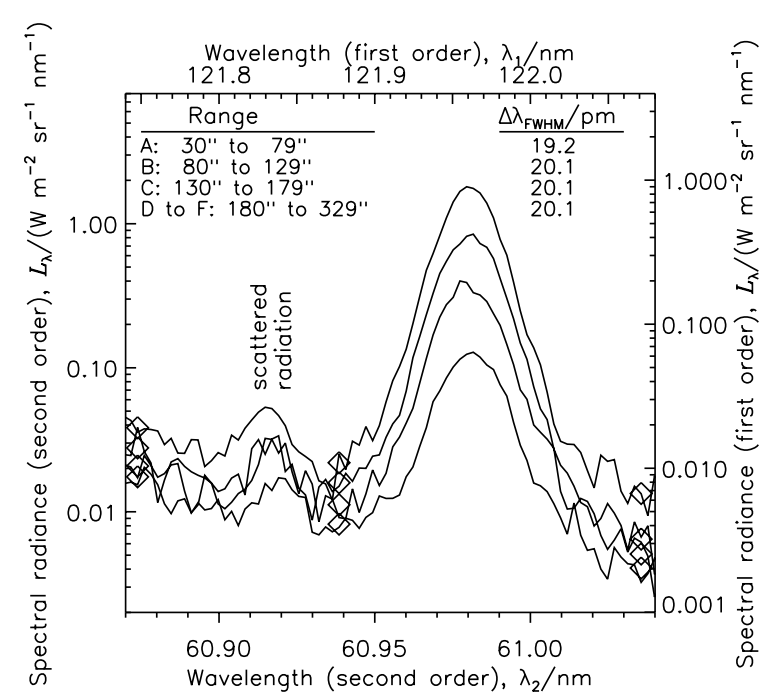

Fig. 4. The spectral radiance in the $\operatorname{Mg} \times 60.98 \mathrm{~nm}$ window in the second order for selected height ranges above a south-polar coronal hole on 16 May 1997. The display is similar to that of Fig. 2b, but the height ranges $\mathrm{D}$ to $\mathrm{F}$ are combined to improve the signal-to-noise ratio. A slit width of $1^{\prime \prime}$ and the bare section of detector B were used during the observations.

The results are presented in Fig. 4 for the $\mathrm{Mg}$ X $60.98 \mathrm{~nm}$ line. The $\Delta \lambda_{\text {FWHM }}$ values lead to corrected Doppler widths, $\Delta \lambda_{\mathrm{D}}$, of $10.8 \mathrm{pm}$ and $11.4 \mathrm{pm}$. The other component of the $\mathrm{Mg} \mathrm{X}$ doublet (not shown) confirmed these findings, even though the profiles became rather noisy at higher altitudes.

\section{Spectroscopic considerations}

Although the SUMER instrument can spectrally resolve most of the lines near the $\mathrm{MgX}$ doublet, there are some lines that hopelessly blend the magnesium lines, as can be seen from Table 1. For the $\mathrm{Mg}$ X $60.98 \mathrm{~nm}$ line, O III and O IV lines have to be taken into account, and for the $62.50 \mathrm{~nm}$ line contributions of P II, Mg II, Si II, and O IV. The relative radiances listed in the third and fourth columns of the table, can help in assessing the importance of the blends. The O III line at $61.00 \mathrm{~nm}$ is weak as it cannot be seen in Fig. 2a on the disk or near the limb. Consequently, the O III $60.97 \mathrm{~nm}$ line is not strong either. The O IV line, on the other hand, contributes $\approx 15 \%$ to the total radiance of $\mathrm{Mg} \mathrm{X}$ and O IV in the height range from $29^{\prime \prime}$ to $80^{\prime \prime}$ (determined by comparing the O IV line at $60.84 \mathrm{~nm}$ with the blend at $60.98 \mathrm{~nm}$ ). To get a value for the disk, the observations of Lemaire et al. (1998) have been considered. Although the OIV line is difficult to separate from the wing of H I Ly $\alpha$, a relative contribution of $\approx 20 \%$ seems to be appropriate. A simulation with Gaussian profiles of equal widths (a worst-case assumption) suggests that this O IV contribution does not significantly influence the $\mathrm{Mg} \mathrm{X}$ width (the relative change will be less than $2 \%$ ). However, it does affect the ratio $L_{62.50} / L_{60.98}$, and a relative contribution of $20 \%$ leads to the observed ratio of 0.4 , provided the $\mathrm{Mg}$ X $62.50 \mathrm{~nm}$ line is not significantly blended. To demonstrate this, we consider again the relative radiances in Table 1 and find that contributions of both the Si II and OIV lines are not very important, with
Table 2. Line widths of the $\operatorname{Mg} X 2{ }^{2} S_{1 / 2}-2 p{ }^{2} P_{1 / 2,3 / 2}$ doublet for selected height ranges in an equatorial region.

\begin{tabular}{ccccc}
\hline \hline Range & \multicolumn{2}{c}{$\Delta \lambda_{\mathrm{FWHM}}^{a} / \mathrm{pm}$} & \multicolumn{2}{c}{$\Delta \lambda_{\mathrm{D}}^{b} / \mathrm{pm}$} \\
$h / \mathrm{Mm}$ & ${ }^{2} \mathrm{P}_{3 / 2}$ & ${ }^{2} \mathrm{P}_{1 / 2}$ & ${ }^{2} \mathrm{P}_{3 / 2}$ & ${ }^{2} P_{1 / 2}$ \\
\hline-45 to -30 & 15.29 & 14.37 & 8.36 & 7.74 \\
-30 to -15 & 16.22 & 14.97 & 8.97 & 8.14 \\
-15 to 0 & 16.67 & 15.01 & 9.26 & 8.17 \\
0 to 15 & 16.69 & 14.93 & 9.27 & 8.12 \\
5 to 30 & 16.86 & 15.23 & 9.38 & 8.32 \\
\hline 21 to 56 & $16.15,15.18$ & $17.05,15.72$ & $7.71^{c}$ & $8.24^{c}$ \\
56 to 91 & $16.56,15.42$ & $17.28,16.36$ & $7.95^{c}$ & $8.55^{c}$ \\
91 to 127 & $17.42,16.63$ & $17.99,17.01$ & $8.70^{c}$ & $9.04^{c}$ \\
127 to 163 & $17.94,16.76$ & $18.51,17.87$ & $8.93^{c}$ & $9.52^{c}$ \\
163 to 198 & $18.11,17.02$ & $18.46,17.87$ & $9.08^{c}$ & $9.50^{c}$ \\
198 to 234 & $19.15,17.36$ & $18.29,18.15$ & $9.56^{c}$ & $9.54^{c}$ \\
\hline
\end{tabular}

${ }^{a}$ Estimated relative standard uncertainty $4 \%$.

${ }^{b}$ A direct conversion of the measured width, $\Delta \lambda_{\mathrm{FWHM}}$, to $\Delta \lambda_{\mathrm{D}}^{*}$ (without corrections for the instrumental width) can be obtained with Eq. (1). It would give, for instance, $\Delta \lambda_{\mathrm{D}}^{*}=9.18 \mathrm{pm}$ and $8.63 \mathrm{pm}$ in the first data row of the table.

${ }^{c}$ Mean value.

possible exceptions near the limb, where the P II and Mg II lines might also have some minor effects. Teriaca et al. (2002) also discussed this wavelength range and concluded that the total blending of the $\operatorname{Mg}$ X $62.50 \mathrm{~nm}$ line on the bare detector of SUMER is not severe.

A comparison of the radiance maps of $\mathrm{Mg}$ X $60.98 \mathrm{~nm}$ and $62.50 \mathrm{~nm}$ obtained during the inter-calibration measurements mentioned in Sect. 1 confirms that a substantial blend of a transition-region line must be present in the $\mathrm{Mg}$ X $60.98 \mathrm{~nm}$ line, because the chromospheric network can clearly be seen in the map of this line, but not in that of the other component of the doublet. With reference to Table 1, the O IV line at $60.98 \mathrm{~nm}$ seems to be the only candidate for such a blend.

Although the blends of CI $124.94 \mathrm{~nm}$ and $\mathrm{Si}$ X $62.47 \mathrm{~nm}$ can be separated from the $\mathrm{Mg} \mathrm{X}$ line, their relative contributions are difficult to assess. On the disk and near the limb the $\mathrm{C} I$ lines appear to be dominant (Fig. 3a), whereas off the limb the Si X is more important (Fig. 3b). Whatever the exact composition may be, the sum cannot significantly influence the width of the $\mathrm{Mg} \mathrm{X}$ line, even at lower spectral resolution. Moreover, the $\mathrm{C}$ I blend is not present in the CDS observations that show $\mathrm{Mg} \mathrm{X}$ and $\mathrm{Si} \mathrm{X}$ in the first order. We, therefore, conclude that the density dependence of the boron-like Si X line cannot be the reason for the narrowing of line width with height observed by CDS.

\section{Discussion}

The results obtained in this study on the $\operatorname{MgX}$ line widths for equatorial regions are summarized in Table 2 and compared with previous results in Fig. 5. We could not include the CDS data, because the half $1 / e$ width, $\Delta \lambda_{\mathrm{D}}^{*}$, plotted in Figs. 3 and 5 of Harrison et al. (2002) are not corrected for the instrumental width and, with $\Delta \lambda_{\mathrm{D}}^{*} / \lambda_{0} \approx 8 \times 10^{-4}$, would be off the scale by more than a factor of four. 


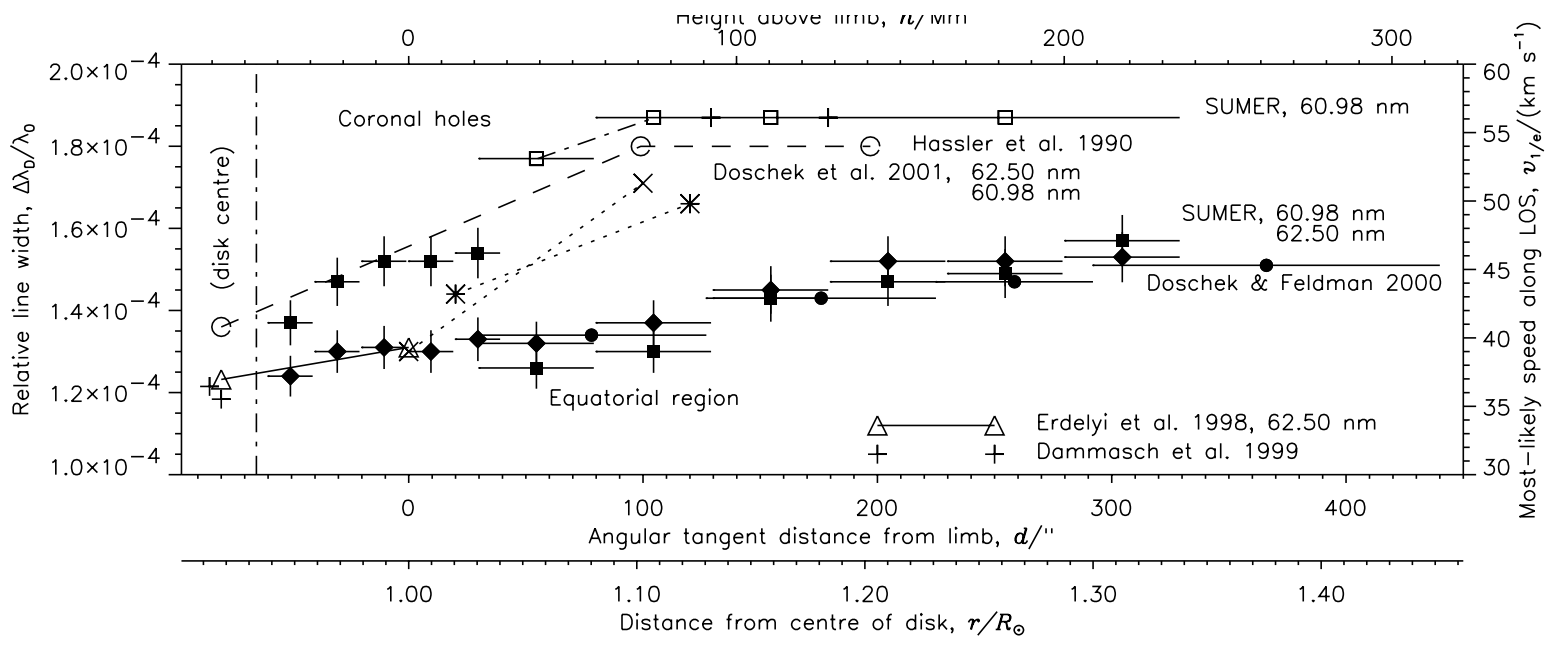

Fig. 5. Relative line-width variations as a function of radial distance from the Sun. Literature data and results obtained in this work (annotated "SUMER") are compiled for the $\mathrm{Mg} \mathrm{X}$ doublet in equatorial and coronal-hole regions. A classification of the Hassler et al. observations, of which only three typical values are shown, is not defined in the 1990 paper. An approximate scale of $715 \mathrm{~km} /{ }^{\prime \prime}$ has been used for both the SOHO observations and those from the Earth. Integration intervals and the SUMER uncertainty margins are marked by horizontal and vertical bars. Related data points are in some cases connected by lines of various styles. They are meant to improve the orientation of the reader, but not as physical interpolations, in particular, for those points representative of centre-of-disk values displayed here near $-80^{\prime \prime}$.

The present results fully confirm the findings of Doschek \& Feldman (2000) deduced from the same observational data set. We have performed, in addition, an uncertainty analysis. The relative standard uncertainty of $\approx 4 \%$ obtained for the line widths implies that there is a small but significant increase of line width with height. In this context, it should be clarified that the relative uncertainty margin of $30 \%$ assumed by Doschek \& Feldman (2000) refers to the calculation of non-thermal velocities and not to the line width as cited by Harrison et al. (2002).

Our results do not indicate a decrease of the width of the $\mathrm{Mg} \mathrm{X}$ lines in the range above $50 \mathrm{Mm}$ up to a height of $\approx 230 \mathrm{Mm}$ for which we have reliable line-width measurements in the equatorial corona in November 1996. Should the narrowing of the $\mathrm{Mg}$ X $62.50 \mathrm{~nm}$ line reported by Harrison et al. (2002) for 2001 near the sunspot maximum be a real effect, the solar conditions in quiet regions of the corona must have changed dramatically with time. In agreement with Harrison and co-workers we are reluctant to accept such a conclusion (cf. Sect. 1), and thus have to question the CDS analysis on the ground that the instrumental width of CDS appears to be too wide to detect variations of the relative line width, $\Delta \lambda_{\mathrm{D}} / \lambda_{0}$, of the order of $1 \times 10^{-4}$. Since no line profiles are displayed for the CDS observations, we do not want to speculate on the reasons for the line-width decreases, and thus are not able to explain the discrepancy with our results, but the observations with much higher spectral resolution obtained by SUMER seem to exclude such a narrowing with altitude under quiet solar conditions.

On the disk, narrow widths of $\Delta \lambda_{\mathrm{D}} \approx 7.4 \mathrm{pm}$ have been measured for both $\mathrm{Mg} \mathrm{X}$ lines in quiet-Sun regions. Our conclusion is that transition-region lines with typical limb brightening factors of four and more (cf. Wilhelm et al. 1998) are only of importance in causing the blends near the limb, and that the mean disk widths of the $\mathrm{Mg} \mathrm{X}$ lines are close to the small value given above. These widths then would be relevant for off-disk scatter considerations. It also means that the values near the limb in Table 2 and Fig. 5 are suspect and probably too wide, in particular for the $60.98 \mathrm{~nm}$ line. Most of the transition-region lines are very weak in the corona, so that no blending is caused by these lines there. If scatter effects would become of importance, the narrow mean disk width of the $\mathrm{Mg} \mathrm{X}$ lines could lead to a decrease of the measured profiles. Considering, however, the relatively constant equatorial scale height of $\approx 50 \mathrm{Mm}$ in Fig. 1 (much smaller than the scatter scale height), scattered radiation is not significant for the SUMER measurements in the low corona.

In Fig. 4, line profiles are shown for several height ranges above a polar coronal hole. The line-width results obtained are also added to the compilation of Fig. 5. The width of the $\operatorname{Mg}$ X $60.98 \mathrm{~nm}$ line increases above the limb. Whether the leveling off near $80 \mathrm{Mm}$ is a real effect cannot be decided unambiguously. Scattered radiation from the solar disk, if present, will tend to reduce the line width, but even with such a contribution, we do not see an indication of a decrease of the line width.

\section{Conclusion}

The line widths of both components of the $\mathrm{Mg} \mathrm{X}$ doublet measured by SUMER monotonically increase in the low corona in equatorial regions in altitude ranges for which scattered radiation from the disk does not play a major rôle. We do not find any evidence for a narrowing of the emission lines above $50 \mathrm{Mm}$. The same statement applies for a coronal hole, but we cannot exclude the possibility of a constant width above $80 \mathrm{Mm}$.

Acknowledgements. The SUMER project is financially supported by the Deutsches Zentrum für Luft- und Raumfahrt (DLR), the Centre National d'Etudes Spatiales (CNES), the National Aeronautics and Space Administration (NASA), and the European Space Agency's (ESA) PRODEX Programme (Swiss contribution). SUMER is part of SOHO, the Solar and Heliospheric Observatory, of ESA and NASA. 


\section{References}

Brueckner, G. E., \& Moe, O. K. 1972, Space Res., XII, 1595 Cohen, L., Feldman, U., \& Doschek, G. A. 1978, ApJS, 37, 393

Curdt, W., Brekke, P., Feldman, U., et al. 2001, A\&A, 375, 591

Dammasch, I. E., Hassler, D. M., Wilhelm, K., \& Curdt, W. 1999, in Proc. of 8th SOHO Workshop: Plasma Dynamics and Diagnostics in the Solar Transition Region and Corona, ed. J.-C. Vial, \& B. Kaldeich-Schürmann (Noordwijk: ESA Publications Division), ESA SP-446, 263

Dere, K. P., Landi, E., Mason, H. E., et al. 1997, A\&AS, 125, 149

Doschek, G. A., \& Feldman, U. 2000, ApJ, 529, 599

Doschek, G. A., Feldman, U., Laming, J. M., et al. 2001, ApJ, 546, 559

Erdélyi, R., Doyle, J. G., Perez, M. E., \& Wilhelm, K. 1998, A\&A, 337,287

Feldman, U., Doschek, G. A., Schühle, U., \& Wilhelm, K. 1999, ApJ, 518,500

Harrison, R. A., Sawyer, E. C., Carter, M. K., et al. 1995, Sol. Phys., 162,233

Harrison, R. A., Hood, A. W., \& Pike, C. D. 2002, A\&A, 392, 319

Hassler, D. M., Rottman, G. J., Shoub, E. C., \& Holzer, T. E. 1990, ApJ, 348, L77
Hassler, D. M., Wilhelm, K., Lemaire, P., \& Schühle, U. 1997, Sol. Phys., 175, 375

Kelly, R. L. 1987, J. Phys. Chem. Ref. Data, 16, 1

Lemaire, P., Wilhelm, K., Curdt, W., et al. 1997, Sol. Phys., 170, 105

Lemaire, P., Emerich, C., Curdt, W., et al. 1998, A\&A, 334, 1095

Mariska, J. T. 1992, The Solar Transition Region (Cambridge: Cambridge University Press)

O'Shea, E., Banerjee, D., \& Poedts, S. 2003, A\&A, 400, 1065

Peter, H., \& Judge, P. G. 1999, ApJ, 522, 1148

Sandlin, G. D., Bartoe, J.-D. F., \& Brueckner, G. E. 1986, ApJS, 61, 801

Seely, J. F., Feldman, U., Schühle, U., et al. 1997, ApJ, 484, L87

Teriaca, L., Madjarska, M. S., \& Doyle, J. G. 2002, A\&A, 392, 309

Teriaca, L., Poletto, G., Romoli, M., \& Biesecker, D. A. 2003, ApJ, 588,566

Wilhelm, K., Curdt, W., Marsch, E., et al. 1995, Sol. Phys., 162, 189

Wilhelm, K., Lemaire, P., Curdt, W., et al. 1997, Sol. Phys., 170, 75

Wilhelm, K., Lemaire, P., Dammasch, I. E., et al. 1998, A\&A, 334, 685

Wilhelm, K., Schühle, U., Curdt, W., et al. 2002, in The Radiometric Calibration of $\mathrm{SOHO}$, ed. A. Pauluhn, M. C. E. Huber, R. v. Steiger (Noordwijk: ISSI/ESA), ISSI Scientific Report, SR-002, 145 